\title{
ANALOG FILTER DIAGNOSIS USING THE OSCILLATION BASED METHOD
}

\author{
Miona Andrejević Stošović — Miljana Milić — Vančo Litovski
}

\begin{abstract}
Oscillation Based Testing (OBT) is an effective and simple solution to the testing problem of continuous time analogue electronic filters. In this paper, diagnosis based on OBT is described for the first time. It will be referred to as OBD. A fault dictionary is created and used to perform diagnosis with artificial neural networks (ANNs) implemented as classifiers. The robustness of the ANN diagnostic concept is also demonstrated by the addition of white noise to the "measured" signals. The implementation of the new concept is demonstrated by testing and diagnosis of a second order notch cell realized with one operational amplifier. Single soft and catastrophic faults are considered in detail and an example of the diagnosis of double soft faults is also given.
\end{abstract}

Ke y w ords: active filters, artificial neural networks, oscillation based diagnosis, oscillation based testing

\section{INTRODUCTION}

One of the fundamental problems in analogue testing is the synthesis of the test signal. The choice is among virtually unlimited possibilities. Analogue testing may be done using DC signals; in the frequency domain; and in the time domain. Of course, if necessary, test signals from several domains may be combined. DC signals usually check for fault effects related to the quiescent conditions and nonlinearities. In the frequency domain one has to find a suitable spectrum for the test signal to activate the fault effect. In the time domain one is to search for one or more signal waveforms to enable testing in the shortest possible time so optimizing the overall production time and decreasing the price of the product [1].

There exists, however, a technique that needs no test signal. It is known as oscillation based testing (OBT) [2]. The basic idea behind this powerful method is to create a redundant feed-back loop that is activated during testing only. By measuring the output signal and by comparing with the response of the fault-free circuit, one can determine whether there are defects in the circuit or not. Note that, instead of creating test signals targeting specific faults, here all possible defects are targeted with only one measurement making OBT very effective.

The implementation of OBT depends on the particular circuit under test because it is necessary to create an oscillator out of the circuit. That is one of the difficulties in implementing the method. In addition, some faults, especially soft ones, create fault effects that are not easily distinguishable. Moreover, while in general the number of test points is reduced to one; the oscillator's output, the problem of measurement is not solved, since one has to decide what parameters of the response are to be extracted. Finally, the method needs to bring the oscillations into a steady state, which frequently slows down the testing process. It is worth mentioning that there are rare situations where no fault effect may be observed when using just one testing point (for example, the output voltage) so additional measurements are needed such as $I_{D D}[3,4]$. That raises the question as to how many test (measurement) points are needed and which quantities are to be extracted to determine the state of the circuit.

Bearing in mind all advantages of the OBT, in this paper we describe the implementation of oscillation based diagnosis (OBD) of analogue circuits. To the best of our knowledge, this is the first time that OBD has been described. The new idea will be demonstrated by implementing the method in a second order notch cell of a discrete analogue active filter. This cell is the most frequently used in the cascade synthesis of active filters. Hence the importance of this case study.

A fault dictionary is created by simulation. It is based on the measurement obtained at just one test point the output terminal. A reduced number of output signal parameters will be extracted. For the implementation, discrete commercial CMOS operational amplifiers will be used. An exhaustive list of single faults and a list of the most probable double faults will be used. The problem of feed-back circuit synthesis will be solved in the simplest manner.

An artificial neural network (ANN) will capture the fault dictionary and perform the diagnosis. The implementation of ANNs for diagnostic purposes will be performed in exactly the same way as was done in $[5,6]$.

\section{THE OSCILLATION BASED TESTING METHOD}

When a suitable feed-back circuit is added to an analogue building block, one may create an oscillator. By

* University of Niš, Faculty of Electronic Engineering, Aleksandra Medvedeva 14, P.O. Box 73 18000 Niš, Serbia (miona.andrejevic, miljana.milic, vanco.litovski)@elfak.ni.ac.rs 
Analog signal processing chain

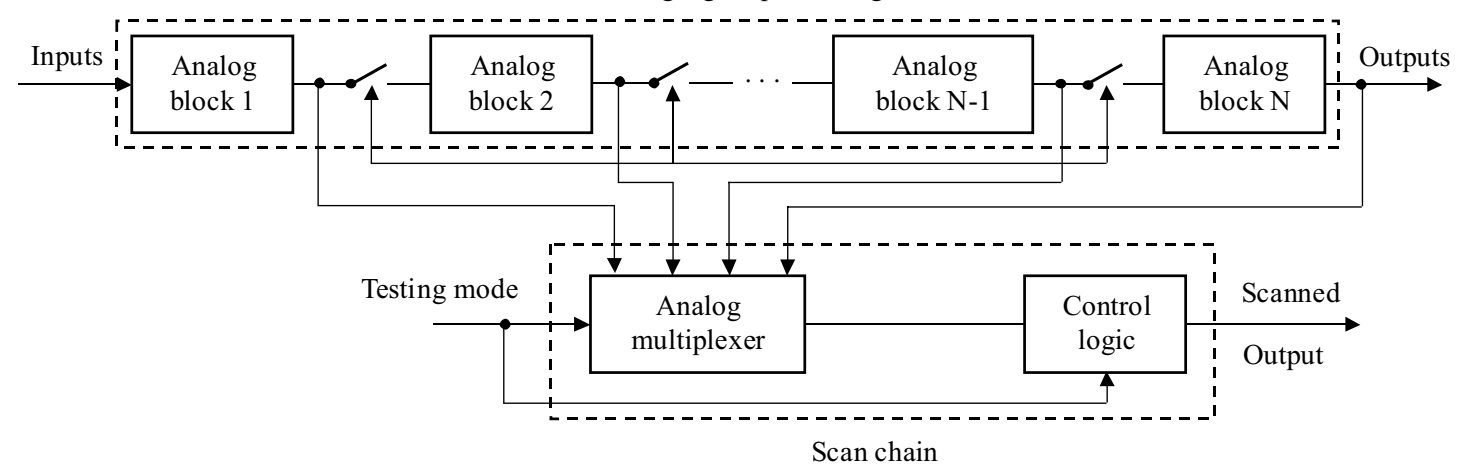

Fig. 1. Architecture of the OBT

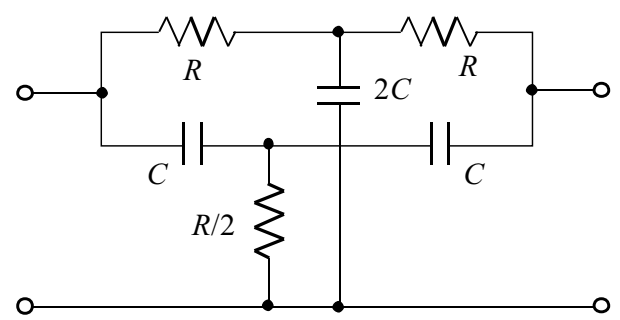

Fig. 2. Twin-T circuit

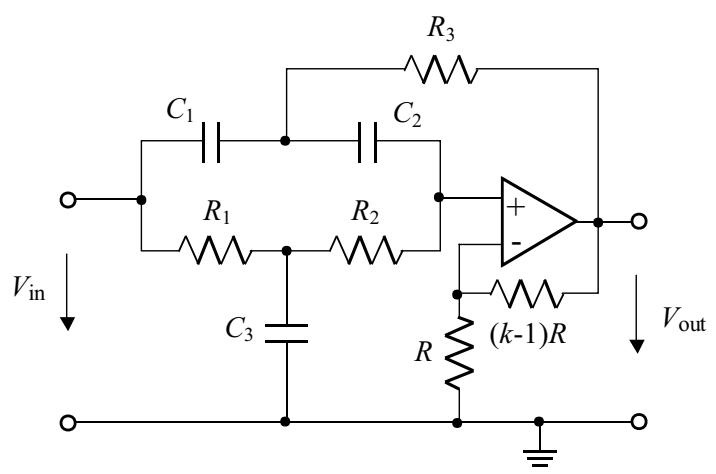

Fig. 3. Sallen-Key (SK) Non-Inverting Second Order Notch Filter Cell

measuring the output voltage (or some other responses) of that circuit one may extract quantities, such as the frequency, the amplitude of the first and other harmonics, the DC value of the output voltage, etc. that may contain information on the presence of a fault in the circuit.

The main advantages of this method are avoiding the search for input stimuli and appropriate mode of operation of the circuit; independence of the method with respect to the type of faults present in the circuit (soft or catastrophic); and simplified selection of the test points and measured quantities at the output [2], [7-9]. From the design for testability (DFT) point of view this method offers exceptional advantages. Namely, by proper structuring of the analogue subsystem on the chip, as shown in Fig. 1, one can control the testing process from outside of the chip.

The idea of this paper is to demonstrate how these defects can be diagnosed using ANNs. This, first, high- lights an important aspect of diagnosis: the number and location of the test points. Simply, we can say that internal test points should be avoided and measurements on the primary inputs and outputs are preferred. This is especially important with DFT concepts such as that depicted in Fig. 1. This is not only related to their automatic observability but also to the nature of diagnostic reasoning. Namely, to diagnose something one looks for functionality, and the function is seen at the primary terminals.

\section{TESTING OF THE NOTCH BIQUAD CELL BY THE OSCILLATION-BASED METHOD}

As a case study for implementation of the oscillation based method, the Sallen-Key non-inverting second order notch filter cell will be used. The importance of this cell comes from the fact that it is the most frequently used cell within every analogue filter, implemented as a cascade of second order cells, irrespective of the position of its passband on the frequency axis. In addition, the specific shape of the transfer function of the feed-back circuit makes the oscillation frequency differ significantly from the notch frequency of the filter cell. The schematic of the cell is based on a twin- $\mathrm{T}$ circuit as shown in Fig. 2, while the actual cell is depicted in Fig. 3 [10].

The circuit in Fig. 2 is a band-stop filter with a notch frequency at $f_{0}=1 /(2 \pi R C)$. It is the basic building block for the active cell shown in Fig. 3, which will be considered here as the circuit under test (CUT). For testing, a positive feed-back loop is created by direct connection of the output to the input of the cell.

The oscillator circuit so obtained is depicted in Fig. 4. The fault-free circuit will oscillate at $f_{N}$. The value of $f_{N}$ will be discussed below. When defects are present one may observe three different situations: a) the faulty circuit oscillates at $f_{N}$, b) the faulty circuit oscillates at some other frequency $\hat{f}_{N}$, and c) there are no oscillations in the circuit. The testing will be successful if there is any difference between the oscillating frequencies and if there are no oscillations. Unfortunately, that is not enough for diagnosis. One needs distinctive information in order to create a diagnostic statement. 


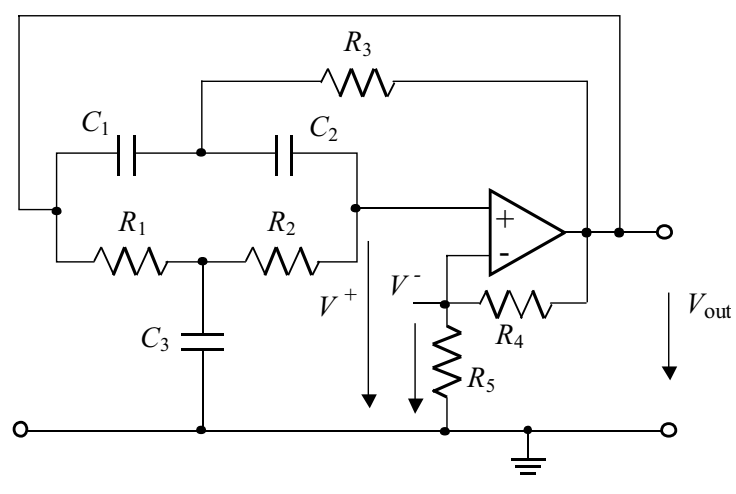

Fig. 4. RC oscillator based on the Notch filter cell

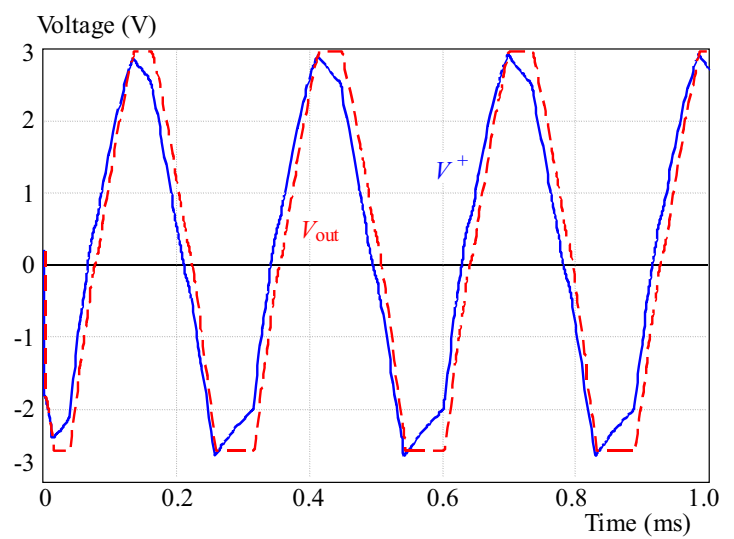

Fig. 5. Responses of the fault free oscillator of Fig. 4 demonstrating the phase shift introduced by the op-amp

The oscillator is usually treated as a linear circuit so one may use the usual oscillator analysis method to get the expected oscillation frequencies. Namely, by writing the modified nodal equations [11] for the circuit of Fig. 4, and equating the system determinant to zero, after separating the real and imaginary parts, one obtains the following two expressions for the possible oscillation frequencies

$$
\begin{array}{r}
\omega_{0}^{2}=(A-1) /\left\{( A - 1 ) C _ { 3 } \left(R_{2} R_{3} C_{2}+R_{1} R_{3} C_{2}+\right.\right. \\
\left.\left.R_{1} R_{2} C_{1}\right)-R_{3} C_{1}\left(R_{2} C_{2}-R_{1} C_{1}\right)\right\}
\end{array}
$$

and

$$
\omega_{0}^{2}=\frac{R_{2} C_{3}+R_{1} C_{3}+R_{3} C_{2}+R_{3} C_{3}-R_{1} C_{1} /(A-1)}{R_{1} R_{2} R_{3} C_{1} C_{2} C_{3}},
$$

where

$$
A=k=1+R_{4} / R_{5} .
$$

One of these expressions is usually required for frequency calculations while the other is needed to find the necessary value of $A$ for sustained oscillation.

The above expressions were derived assuming that the operational amplifiers is ideal with the infinite gain, which is not true in a real circuit. This is important since the closed-loop-gain of the oscillator circuit is characterized by both modulus and phase. Both are frequency dependent and fundamentally determine the oscillation frequency. The operational amplifier's phase shift becomes of major importance in this situation and has to be taken into account. So, the expressions given by (1) or (2), may be used as reference only. This will be confirmed below after simulation experiments are shown. It is worth mentioning that the need to include the operational amplifier's phase shift has been previously noted [10].

The fault list used contains both parametric (soft) and catastrophic faults in the passive feedback circuit.

When testing or diagnosing a circuit one needs to create a fault dictionary - that is a table containing faults and fault effects. To build it, a large number (equal to the number of modelled defects) of repetitive simulations has to be performed. This is known as "simulation before test". For every simulation, a fault is inserted in the original oscillator circuit, so creating a new oscillator. Note that simulation of an oscillator is not a straightforward task since one usually uses an A-stable integration rule (such as Euler-backward) for solving the differential equations of the circuit, in order to the prevent oscillations in the simulation. Here however, since the circuit is unstable, one needs to use an integration rule that is not A-stable (trapezoidal, for example) [11]. Hence, the simulation can model oscillations. For correct simulation one needs to use a complete schematic of the operational amplifier or a qualified model that performs well from the phase-shift point of view. To illustrate how important the modelling of the phase difference inserted by the operational amplifier is, the simulation results for a fault free circuit (oscillator) are depicted in Fig. 5. For the operational amplifier, a SPICE model taken from the component's datasheet was used [12]. Two signals are shown, the operational amplifier's input (non-inverting terminal) and its output. One can deduce from the figure a phase shift of $12.5^{\circ}$. This is to be compared to the maximum phase shift of the feedback circuit which is $16.7^{\circ}$ [13] meaning that a significant difference between the oscillation frequency $f_{N}$ and that given by (1) may be expected.

\section{CONCEPTS OF DIAGNOSIS}

Defects that will be modelled in the following example are categorized into several groups:

- Catastrophic defects within the RC circuit.

- Parametric defects within the RC circuit.

- Separate examples of multiple parametric defects within the RC circuit.

A table containing a set of faults and the corresponding responses or parameters extracted from the responses of the system is referred to as fault dictionary. This is important not only for testing but also for diagnostic purposes. Since the number of possible faults in a system may be very large, when creating the fault dictionary one generally chooses a set of the most probable faults. If this approach is implemented, no faults will be diagnosed except the ones conceived in advance and included in the fault list. 
Table 1. Catastrophic defects (SC stands for short circuit, OP stands for open circuit, NO stands for no oscillations)

\begin{tabular}{cccc}
\hline No. & $\begin{array}{c}\text { Defect } \\
\text { type }\end{array}$ & $\begin{array}{c}\text { Element } \\
\text { value }\end{array}$ & $\begin{array}{c}\text { Oscillating frequency }(\mathrm{kHz}) \\
\text { /relative increment }(\%)\end{array}$ \\
\hline 1 & Fault free & & $3.48 / 0.0$ \\
2 & SC: $R_{1}$ & $R_{1}=0 \Omega$ & $\mathrm{NO}$ \\
3 & SC: $R_{2}$ & $R_{2}=0 \Omega$ & $\mathrm{NO}$ \\
4 & SC: $R_{3}$ & $R_{3}=0 \Omega$ & $2.12 /-39.08$ \\
5 & OP: $R_{1}$ & $R_{1}=\infty \Omega$ & $3.14 /-9.77$ \\
6 & OP: $R_{2}$ & $R_{2}=\infty \Omega$ & $\mathrm{NO}$ \\
7 & OP: $R_{3}$ & $R_{3}=\infty \Omega$ & $3.32 /-4.6$ \\
8 & SC: $C_{1}$ & $C_{1}=\infty \mathrm{F}$ & $2.76 /-20.69$ \\
9 & SC: $C_{2}$ & $C_{2}=\infty \mathrm{F}$ & $2.12 /-39.08$ \\
10 & SC: $C_{3}$ & $C_{3}=\infty \mathrm{F}$ & $2.4 /-31.03$ \\
11 & OP: $C_{1}$ & $C_{1}=0 \mathrm{~F}$ & $\mathrm{NO}$ \\
12 & OP: $C_{2}$ & $C_{2}=0 \mathrm{~F}$ & $\mathrm{NO}$ \\
13 & OP: $C_{3}$ & $C_{3}=0 \mathrm{~F}$ & $\mathrm{NO}$ \\
\hline
\end{tabular}

Table 2. Parametric defects

\begin{tabular}{ccc}
\hline No. & $\begin{array}{c}\text { Defect } \\
\text { type }\end{array}$ & $\begin{array}{c}\text { Oscillating frequency }(\mathrm{kHz}) \\
\text { /relative increment }(\%)\end{array}$ \\
\hline 1 & $1.2 \cdot R_{1}$ & $3.44 /-1.15$ \\
2 & $0.8 \cdot R_{1}$ & $3.54 / 1.72$ \\
3 & $1.2 \cdot R_{2}$ & $3.1 /-10.92$ \\
4 & $0.8 \cdot R_{2}$ & $4.02 / 15.52$ \\
5 & $1.2 \cdot R_{3}$ & $3.46 /-0.57$ \\
6 & $0.8 \cdot R_{3}$ & $3.5 / 0.57$ \\
7 & $1.2 \cdot C_{1}$ & $3.38 /-2.87$ \\
8 & $0.8 \cdot C_{1}$ & $3.62 / 4.02$ \\
9 & $1.2 \cdot C_{2}$ & $3.28 /-5.75$ \\
10 & $0.8 \cdot C_{2}$ & $3.8 / 9.2$ \\
11 & $1.2 \cdot C_{3}$ & $3.32 /-4.6$ \\
12 & $0.8 \cdot C_{3}$ & $3.7 / 6.32$ \\
\hline
\end{tabular}

Table 3. Two defects present simultaneously

\begin{tabular}{ccc}
\hline No. & $\begin{array}{c}\text { Defect } \\
\text { type }\end{array}$ & $\begin{array}{c}\text { Oscillating frequency }(\mathrm{kHz}) \\
\text { /relative increment }(\%)\end{array}$ \\
\hline 1 & $1.2 \cdot C_{2} ; 1.2 \cdot C_{3}$ & $3.1 /-10.92$ \\
2 & $0.8 \cdot C_{2} ; 0.8 \cdot C_{3}$ & $4 / 14.94$ \\
3 & $1.2 \cdot C_{2} ; 0.8 \cdot C_{3}$ & $3.52 / 1.15$ \\
4 & $0.8 \cdot C_{2} ; 1.2 \cdot C_{3}$ & $3.64 / 4.6$ \\
5 & $1.2 \cdot R_{1} ; 1.2 \cdot C_{1}$ & $3.34 /-4.02$ \\
6 & $0.8 \cdot R_{1} ; 0.8 \cdot C_{1}$ & $3.7 / 6.32$ \\
7 & $1.2 \cdot R_{1} ; 0.8 \cdot C_{1}$ & $3.56 / 2.3$ \\
8 & $0.8 \cdot R_{1} ; 1.2 \cdot C_{1}$ & $3.46 /-0.57$ \\
9 & $1.2 \cdot R_{2} ; 1.2 \cdot C_{2}$ & $2.9 /-16.67$ \\
10 & $0.8 \cdot R_{2} ; 0.8 \cdot C_{2}$ & $4.34 / 24.71$ \\
11 & $1.2 \cdot R_{2} ; 0.8 \cdot C_{2}$ & $3.38 /-2.87$ \\
12 & $0.8 \cdot R_{2} ; 1.2 \cdot C_{2}$ & $3.78 / 8.62$ \\
\hline
\end{tabular}

Searching through the fault dictionary, in general, enables the fault coverage to be established and the test signal to be validated. In addition, by applying a specific inference mechanism, after the search, one may create a qualified diagnostic statement.

The actual process of generating the final diagnostic statement may be based on different concepts. When combinational digital circuits are diagnosed one usually creates fault tables and searches them to locate the fault. Localization of faults in sequential digital circuits is performed by edge-pin testing or by guided-probe testing [14]. Due to the diversity of the stimuli and to different domains to be considered, analogue diagnosis has been covered by many approaches, as described in detail in [5]. Thanks to the advances in computational intelligence in recent decades, new diagnostic paradigms have been applied based on: model-based concepts [15]; productionrule based artificial intelligence [16,17]; ANNs [18]; genetic algorithms [19]; and fuzzy-reasoning [20]; all trying to create an approach that exhibits properties that we might consider to be "intelligent behavior". A comprehensive overview of the complete subject of diagnosis of analog electronic circuits may be found in [5].

In our approach, artificial neural networks were applied to the diagnosis of analogue and mixed signal circuits $[5,6]$. We claim here that ANNs, being universal approximators [21], are the best way both to capture the mapping, and to reproduce the fault code based on measured data, performing classification per se, and thereby performing diagnosis. If a large number of faults and a reduced number of outputs are to be conceived in the same time, thanks to the resemblance of the fault effects, the search process within the fault dictionary requires highly sophisticated classification algorithms.

\section{FAULT SIMULATION AND FAULT DICTIONARY CREATION}

For every passive element within the RC circuit (Fig. 4), a short- and open circuit is considered as a catastrophic fault. After insertion, the resulting schematic was simulated and the results are shown in Table 1 . The resistors in the negative feed-back circuit were chosen to be $R_{4}=2 \mathrm{k} \Omega$, and $R_{5}=20 \mathrm{k} \Omega$, leading to a gain $A=1.1$. The operational amplifier used in the simulation is the LTC6078.

By inspection of Table 1 we find that in six out of twelve cases the circuit keeps oscillating. In all the cases the fault effect is recognizable, because the oscillation frequency is never the same as that of the fault-free circuit. In general, however, one may state that by implementing OBT we got almost perfect fault coverage of catastrophic faults. We also calculated the frequency increment relative to the oscillating frequency, and it is given in $\%$. The minus (-) sign stands for the cases where the absolute value of the oscillating frequency is less than in the case of the fault-free circuit, and for the rest of the cases, the relative increment is positive.

When considering parametric defects one has to decide to what extent a change of the element value is to be 
Table 4. The fault dictionary

\begin{tabular}{cccc}
\hline No. & $\begin{array}{c}\text { Defect } \\
\text { type }\end{array}$ & $\begin{array}{c}\text { Oscillating } \\
\text { frequency }(\mathrm{kHz})\end{array}$ & $\mathrm{THD}(\%)$ \\
\hline 0 & $\mathrm{Fault}$ free & 3.48 & 44.25 \\
1 & $\mathrm{SC}: R_{1}$ & $\mathrm{NO}$ & $\mathrm{NO}$ \\
2 & $\mathrm{SC}: R_{2}$ & $\mathrm{NO}$ & $\mathrm{NO}$ \\
3 & $\mathrm{SC}: R_{3}$ & 2.12 & 67.09 \\
4 & $\mathrm{OP}: R_{1}$ & 3.14 & 9.826 \\
5 & $\mathrm{OP}: R_{2}$ & $\mathrm{NO}$ & $\mathrm{NO}$ \\
6 & $\mathrm{OP}: R_{3}$ & 3.32 & 26.85 \\
7 & $\mathrm{SC}: C_{1}$ & 2.76 & 12.443 \\
8 & $\mathrm{SC}: C_{2}$ & 2.12 & 67.09 \\
9 & $\mathrm{SC}: C_{3}$ & 2.4 & 46.44 \\
10 & $\mathrm{OP}: C_{1}$ & $\mathrm{NO}$ & $\mathrm{NO}$ \\
11 & $\mathrm{OP}: C_{2}$ & $\mathrm{NO}$ & $\mathrm{NO}$ \\
12 & $\mathrm{OP}: C_{3}$ & $\mathrm{NO}$ & $\mathrm{NO}$ \\
13 & $1.2 \cdot R_{1}$ & 3.44 & 33.555 \\
14 & $0.8 \cdot R_{1}$ & 3.54 & 40.877 \\
15 & $1.2 \cdot R_{2}$ & 3.1 & 17.701 \\
16 & $0.8 \cdot R_{2}$ & 4.02 & 23.108 \\
17 & $1.2 \cdot R_{3}$ & 3.46 & 26.902 \\
18 & $0.8 \cdot R_{3}$ & 3.5 & 48.13 \\
19 & $1.2 \cdot C_{1}$ & 3.38 & 30.56 \\
20 & $0.8 \cdot C_{1}$ & 3.62 & 53.35 \\
21 & $1.2 \cdot C_{2}$ & 3.28 & 30.256 \\
22 & $0.8 \cdot C_{2}$ & 3.8 & 24.3 \\
23 & $1.2 \cdot C_{3}$ & 3.32 & 34.23 \\
24 & $0.8 \cdot C_{3}$ & 3.7 & 26.93 \\
25 & $1.2 \cdot C_{2} ; 1.2 \cdot C_{3}$ & 3.1 & 23.59 \\
26 & $0.8 \cdot C_{2} ; 0.8 \cdot C_{3}$ & 4 & 28.812 \\
27 & $1.2 \cdot C_{2} ; 0.8 \cdot C_{3}$ & 3.52 & 39.144 \\
28 & $0.8 \cdot C_{2} ; 1.2 \cdot C_{3}$ & 3.64 & 28.7 \\
29 & $1.2 \cdot R_{1} ; 1.2 \cdot C_{1}$ & 3.34 & 19.28 \\
30 & $0.8 \cdot R_{1} ; 0.8 \cdot C_{1}$ & 3.7 & 51.97 \\
31 & $1.2 \cdot R_{1} ; 0.8 \cdot C_{1}$ & 3.56 & 26.45 \\
32 & $0.8 \cdot R_{1} ; 1.2 \cdot C_{1}$ & 3.46 & 23.75 \\
33 & $1.2 \cdot R_{2} ; 1.2 \cdot C_{2}$ & 2.9 & 25.2 \\
34 & $0.8 \cdot R_{2} ; 0.8 \cdot C_{2}$ & 4.34 & 17.476 \\
35 & $1.2 \cdot R_{2} ; 0.8 \cdot C_{2}$ & 3.38 & 28.65 \\
36 & $0.8 \cdot R_{2} ; 1.2 \cdot C_{2}$ & 3.78 & 28.461 \\
\hline & & &
\end{tabular}

Table 5. Ambiguity groups

\begin{tabular}{cccc}
\hline $\begin{array}{c}\text { Ambiguity } \\
\text { group }\end{array}$ & $\begin{array}{c}\text { Faults } \\
\text { included }\end{array}$ & $\begin{array}{c}\text { Oscillating } \\
\text { frequency }(\mathrm{kHz})\end{array}$ & $\begin{array}{c}\text { THD } \\
(\%)\end{array}$ \\
\hline \multirow{4}{*}{ AG1 $R_{1}$} & & \\
& SC: $R_{2}$ & & \\
& OP: $R_{2}$ & NO & NO \\
& OP: $C_{1}$ & & \\
& OP: $C_{2}$ & & \\
& OP: $C_{3}$ & & \\
\hline \multirow{2}{*}{ AG2 } & SC: $C_{2}$ & \multirow{2}{*}{2.12} & 67.09 \\
& SC: $R_{3}$ & & \\
\hline
\end{tabular}

treated as a defect having in mind that small changes may be looked upon as tolerances coming from the technology process. Here a parametric defect will be seen when the element value within the RC-circuit is changed by $20 \%$ in comparison to its nominal value. Both positive and negative changes are taken into account. As a result Table 2 is produced. It contains the faults and the fault effects for all modelled soft faults in the RC-circuit. By inspection of Table 2 one may easily conclude that in all cases there is difference between the behavior of the fault-free and the faulty circuit. In six cases the change in the frequency value is relatively low (less than $5 \%$ ) so making the decision difficult. Here again we may conclude that the fault coverage is almost perfect. The possible number of double soft defects is much larger than in the case of the single faults. This is why a reduced set of pairs of soft faults was considered as shown in Table 3. One may observe that in all cases the faulty circuit exhibits a new value for $f_{N}$. In six cases the change in the frequency value is also small.

In summary, considering the OBT approach applied to the notch filter cell, we have to observe the following. First of all, no test signal was needed. That is a big advantage to the test engineer. Second, only one test point was observed, the circuit's output, which is the most natural point of access for measurement. Finally, only one quantity was extracted as a measure of fault coverage: the oscillation frequency. With such a simple procedure a large number of faults was covered, leading to the conclusion that the OBT method, for this example, is an excellent testing concept.

By inspection of Tables 1-3, one may find that some of the fault effects, while different from the fault-free response, are identical to each other. We refer to these types of faults as ambiguity groups or functionally equivalent faults (FEF) [22]. These groups do not affect the testing since the go-no-go concept is applied, ie the information that there is a fault in the system is enough to decide whether the circuit should be discarded. In cases where diagnosis is to be performed, however, FEF limits the diagnostic resolution, so one should try to keep the number of FEFs to a minimum.

\section{FAULT DIAGNOSIS}

Following these considerations we needed to find some additional quantity to support the creation of the diagnostic statement. Using an additional test point or even redesigning the circuit to create the possibility for monitoring the supply current, as was proposed in [3] and [4], we consider to be unacceptably expensive and inconvenient for application in DFT designs. Instead we were looking for a parameter that can be extracted from the same measurement. Furthermore, we would prefer to implement the same parameter extraction procedure for the new parameter as that used for the extraction of the oscillation frequency. So, keeping the circuits output as the only measured quantity and after performing spectral analysis to get the frequency it became natural to use the total harmonic distortion (THD) of the output signal as the additional information for diagnosis. 
Table 6. The fault dictionary used for ANN training

\begin{tabular}{|c|c|c|c|c|}
\hline No. & $\begin{array}{l}\text { Defect } \\
\text { type }\end{array}$ & $\begin{array}{c}\text { Oscillating } \\
\text { frequency }(\mathrm{kHz}) \\
\text { /relative } \\
\text { increment }(\%)\end{array}$ & $\operatorname{THD}(\%)$ & $\begin{array}{l}\text { Fault } \\
\text { code }\end{array}$ \\
\hline 0 & Fault free & $3.48 / 0$ & 44.25 & 0 \\
\hline 1 & AG1 & $5 / 43.68$ & 70 & 3 \\
\hline 2 & AG2 & $2.12 /-39.08$ & 67.09 & -11 \\
\hline 3 & $\mathrm{OP}: R_{1}$ & $3.14 /-9.77$ & 9.826 & 12 \\
\hline 4 & $\mathrm{OP}: R_{3}$ & $3.32 /-4.6$ & 26.85 & 5 \\
\hline 5 & $\mathrm{SC}: C_{1}$ & $2.76 /-20.69$ & 12.443 & -8 \\
\hline 6 & $\mathrm{SC}: C_{3}$ & $2.4 /-31.03$ & 46.44 & 8 \\
\hline 7 & $1.2 \cdot R_{1}$ & $3.44 /-1.15$ & 33.555 & 9 \\
\hline 8 & $0.8 \cdot R_{1}$ & $3.54 / 1.72$ & 40.877 & -2 \\
\hline 9 & $1.2 \cdot R_{2}$ & $3.1 /-10.92$ & 17.701 & 1 \\
\hline 10 & $0.8 \cdot R_{2}$ & $4.02 / 15.52$ & 23.108 & -12 \\
\hline 11 & $1.2 \cdot R_{3}$ & $3.46 /-0.57$ & 26.902 & 2 \\
\hline 12 & $0.8 \cdot R_{3}$ & $3.5 / 0.57$ & 48.13 & 11 \\
\hline 13 & $1.2 \cdot C_{1}$ & $3.38 /-2.87$ & 30.56 & 6 \\
\hline 14 & $0.8 \cdot C_{1}$ & $3.62 / 4.02$ & 53.35 & -9 \\
\hline 15 & $1.2 \cdot C_{2}$ & $3.28 /-5.75$ & 30.256 & -5 \\
\hline 16 & $0.8 \cdot C_{2}$ & $3.8 / 9.2$ & 24 & 14 \\
\hline 17 & $1.2 \cdot C_{3}$ & $3.32 /-4.6$ & 34.23 & -13 \\
\hline 18 & $0.8 \cdot C_{3}$ & $3.7 / 6.32$ & 26.93 & -3 \\
\hline 19 & $1.2 \cdot C_{2} ; 1.2 \cdot C_{3}$ & $3.1 /-10.92$ & 23.59 & -1 \\
\hline 20 & $0.8 \cdot C_{2} ; 0.8 \cdot C_{3}$ & 4/14.94 & 28.812 & 7 \\
\hline 21 & $1.2 \cdot C_{2} ; 0.8 \cdot C_{3}$ & $3.52 / 1.15$ & 39.144 & -4 \\
\hline 22 & $0.8 \cdot C_{2} ; 1.2 \cdot C_{3}$ & $3.64 / 4.6$ & 28.7 & -15 \\
\hline 23 & $1.2 \cdot R_{1} ; 1.2 \cdot C_{1}$ & $3.34 /-4.02$ & 19.28 & -7 \\
\hline 24 & $0.8 \cdot R_{1} ; 0.8 \cdot C_{1}$ & $3.7 / 6.32$ & 51.97 & 13 \\
\hline 25 & $1.2 \cdot R_{1} ; 0.8 \cdot C_{1}$ & $3.56 / 2.3$ & 26.45 & 15 \\
\hline 26 & $0.8 \cdot R_{1} ; 1.2 \cdot C_{1}$ & $3.46 /-0.57$ & 23.75 & -14 \\
\hline 27 & $1.2 \cdot R_{2} ; 1.2 \cdot C_{2}$ & $2.9 /-16.67$ & 25.2 & 10 \\
\hline 28 & $0.8 \cdot R_{2} ; 0.8 \cdot C_{2}$ & $4.34 / 24.71$ & 17.476 & 4 \\
\hline 29 & $1.2 \cdot R_{2} ; 0.8 \cdot C_{2}$ & $3.38 /-2.87$ & 28.65 & -10 \\
\hline 30 & $0.8 \cdot R_{2} ; 1.2 \cdot C_{2}$ & $3.78 / 8.62$ & 28.461 & -6 \\
\hline
\end{tabular}

For diagnostic purpose we created a merged fault dictionary (Table 4) including defects from Tables 1, 2, 3, respectively. A column was added, however, containing the values of THD. From Table 4 we can deduce that, in the vast majority of cases, the values of THD differ from each other, so diagnosis is possible. One can find from the same Table, however, that some defects have the same values for both the oscillating frequency and THD (defects No. 1, No. 2, No. 5, No. 10, No. 11, No. 12, and also defects No. 3 and No. 8) meaning that we still have FEFs. These are extracted from Table 4 and reproduced in Table 5. The new ambiguity groups are denoted as AG1 and AG2. For the case of AG1, where there were no oscillations in the circuit, it was not possible to calculate THD (denoted as NO in the Table 4).

The presence of these ambiguity groups introduces minor changes into the fault dictionary from the Table 4, since only one representative of each ambiguity group was included in the fault dictionary. Table 6 gives the final version of the dictionary, later used for the diagnosis. Since, even for this case, we needed to assign some concrete values that would be used in the ANN training procedure, we chose values $5 \mathrm{kHz}$ for the frequency and $70 \%$ for THD, as the greatest parameter values for these groups.

The defect's coding is presented in the last column of Table 6 by: Fault code $(m)$. This version of the fault dictionary is ready for training the ANN that will later perform the diagnosis.

The fault coding is an important issue. In fact, some defects exhibit very similar effects. Namely, input data (signatures) can have very close numerical values, and if the output values (defect codes) were also similar, the network could not always be trained successfully. For example, we notice that defects Nos. 9 and 19, Nos. 4 and 17, Nos. 13 and 29, Nos. 11 and 26, Nos. 18 and 24 have the same values for the oscillating frequency and very close values for THD, and hence we assigned codes that are not close, $(1$ and $-15,5$ and $-13,6$ and $-10,2$ and $-14,-3$ and 13 , respectively), in order to differentiate them. Fault code $m=0$ denotes the fault free circuit, and codes are in the interval $[-15,15]$. In general, faults are coded randomly, so that faults with similar effects are unlikely to have similar codes, and if that happens, the codes are reordered in order to make a bigger distinction. This approach has proven to be good, because the coding influenced the training time of the $\mathrm{ANN}$, and also, the training error.

For diagnosis, fully connected feed-forward ANNs with sigmoidal activation function, for the neurons in the hidden layer, and with a linear function, for the neurons in the output layer, were used. With two pieces of data for each fault, the neural network input structure was restricted to two input terminals. The relative increment (\%) was the first ANN input. We chose the increment instead of the oscillating frequency because these data have a wider range, $[-39.08,43.68]$, so being numerically easier to discern while the oscillating frequency is in the range $[2.12,5]$. This allows the ANN to be trained to learn the fault code more successfully with a smaller number of neurons in the hidden layer. The THD is the second input. The ANN learns the fault code.

In the exploitation phase, when excited by the measured relative increment of the oscillation frequency and the THD values, the ANN diagnoses the fault by outputting the fault-code as a signal level, so we needed only one output neuron. The number of hidden neurons $n$, was found by trial and error after several iterations starting with an estimation based on that in [23]. The goal was to find the optimum $n$ that leads to a satisfactory classification. Using too many neurons would increase the training time, but using too few would starve the network of the resources needed to solve the problem. Also, an excessive number of hidden neurons may cause the over-fitting problem [24]. In practice, 20 hidden neurons were used. 
Table 7. ANN responses

\begin{tabular}{cccc}
\hline No. & Defect type & $\begin{array}{c}\text { Fault code } \\
\text { (expected) }\end{array}$ & $\begin{array}{c}\text { ANN } \\
\text { response }\end{array}$ \\
\hline 0 & Fault free & 0 & 0.00023 \\
1 & SC: $R_{1}$ & 3 & 2.98743 \\
2 & SC: $R_{3}$ & -11 & -11.0297 \\
3 & OP: $R_{1}$ & 12 & 12.0047 \\
4 & OP: $R_{3}$ & 5 & 5.00297 \\
5 & SC: $C_{1}$ & -8 & -7.92505 \\
6 & SC: $C_{3}$ & 8 & 7.99517 \\
7 & $1.2 \cdot R_{1}$ & 9 & 9.00308 \\
8 & $0.8 \cdot R_{1}$ & -2 & -1.78515 \\
9 & $1.2 \cdot R_{2}$ & 1 & 1.01037 \\
10 & $0.8 \cdot R_{2}$ & -12 & -11.9991 \\
11 & $1.2 \cdot R_{3}$ & 2 & 2.0044 \\
12 & $0.8 \cdot R_{3}$ & 11 & 11 \\
13 & $1.2 \cdot C_{1}$ & 6 & 6.00198 \\
14 & $0.8 \cdot C_{1}$ & -9 & -9.00061 \\
15 & $1.2 \cdot C_{2}$ & -5 & -4.9262 \\
16 & $0.8 \cdot C_{2}$ & 14 & 14.0097 \\
17 & $1.2 \cdot C_{3}$ & -13 & -12.9816 \\
18 & $0.8 \cdot C_{3}$ & -3 & -2.98707 \\
19 & $0.8 \cdot C_{2} ; 1.2 \cdot C_{3}$ & -15 & -14.9913 \\
20 & $0.8 \cdot C_{2} ; 0.8 \cdot C_{3}$ & 7 & 7.00478 \\
21 & $1.2 \cdot C_{2} ; 0.8 \cdot C_{3}$ & -4 & -3.74645 \\
22 & $1.2 \cdot C_{2} ; 1.2 \cdot C_{3}$ & -1 & -0.970082 \\
23 & $1.2 \cdot R_{1} ; 1.2 \cdot C_{1}$ & -7 & -6.98762 \\
24 & $0.8 \cdot R_{1} ; 0.8 \cdot C_{1}$ & 13 & 13.0002 \\
25 & $1.2 \cdot R_{1} ; 0.8 \cdot C_{1}$ & 15 & 15.008 \\
26 & $0.8 \cdot R_{1} ; 1.2 \cdot C_{1}$ & -14 & -13.9907 \\
27 & $1.2 \cdot R_{2} ; 1.2 \cdot C_{2}$ & 10 & 10.0079 \\
28 & $0.8 \cdot R_{2} ; 0.8 \cdot C_{2}$ & 4 & 3.9985 \\
29 & $1.2 \cdot R_{2} ; 0.8 \cdot C_{2}$ & -10 & -9.99773 \\
30 & $0.8 \cdot R_{2} ; 1.2 \cdot C_{2}$ & -6 & -5.98843 \\
\hline & & &
\end{tabular}

The effectiveness of the training process of the obtained ANN was verified by exciting the ANN with faulty inputs. The responses of the ANN show that there were no errors in classifying the faults as is presented in Table 7 . The ANN response was considered to be correct when its value was in the range $[(m-0.5),(m+0.5)]$. After successful training, no mistakes were observed for all 30 faults.

Further validation of the model was needed since noise is always present when measurement is performed. We simulated this situation by adding white noise, which plays the role of the voltmeter measurement error, in series to the circuit output. The white noise amplitude was chosen to be $1 \%$ of the signal amplitude. The simulation results show that white noise does not change the oscillating frequency at all, but it changes the THD. These changes are presented in Table 8. THD in the third column of the Table is for the ideal case, with no error (as was presented earlier in the text), and $\mathrm{THD}_{N}$ in the fourth column is calculated after the simulation with white noise. In the last column of the Table we present the relative THD increment. These data show that this increment is in all cases smaller than $1 \%$. We can con-
Table 8. THD changes due to the measurement error

\begin{tabular}{|c|c|c|c|c|}
\hline No. & Defect type & $\begin{array}{c}\text { THD } \\
\%\end{array}$ & $\underset{\%}{\mathrm{THD}_{\mathrm{N}}}$ & $\begin{array}{c}\text { Relative } \\
\text { increment in } \%\end{array}$ \\
\hline 0 & Fault free & 44.25 & 44.16 & 0.2 \\
\hline 1 & AG1 & $\mathrm{NO}$ & $\mathrm{NO}$ & / \\
\hline 2 & AG2 & 67.09 & 67.06 & 0.04 \\
\hline 3 & $\mathrm{OP}: R_{1}$ & 9.826 & 9.829 & 0.03 \\
\hline 4 & $\mathrm{OP}: R_{3}$ & 26.85 & 26.63 & 0.82 \\
\hline 5 & $\mathrm{SC}: C_{1}$ & 12.443 & 12.438 & 0.04 \\
\hline 6 & $\mathrm{SC}: C_{3}$ & 46.44 & 46.64 & 0.43 \\
\hline 7 & $1.2 \cdot R_{1}$ & 33.555 & 33.438 & 0.35 \\
\hline 8 & $0.8 \cdot R_{1}$ & 40.877 & 40.813 & 0.16 \\
\hline 9 & $1.2 \cdot R_{2}$ & 17.701 & 17.669 & 0.18 \\
\hline 10 & $0.8 \cdot R_{2}$ & 23.108 & 23.236 & 0.55 \\
\hline 11 & $1.2 \cdot R_{3}$ & 26.902 & 26.893 & 0.03 \\
\hline 12 & $0.8 \cdot R_{3}$ & 48.13 & 48.06 & 0.15 \\
\hline 13 & $1.2 \cdot C_{1}$ & 30.56 & 30.64 & 0.26 \\
\hline 14 & $0.8 \cdot C_{1}$ & 53.35 & 53.33 & 0.04 \\
\hline 15 & $1.2 \cdot C_{2}$ & 30.256 & 30.425 & 0.56 \\
\hline 16 & $0.8 \cdot C_{2}$ & 24.3 & 24.095 & 0.84 \\
\hline 17 & $1.2 \cdot C_{3}$ & 34.23 & 34.182 & 0.14 \\
\hline 18 & $0.8 \cdot C_{3}$ & 26.93 & 26.83 & 0.37 \\
\hline 19 & $1.2 \cdot C_{2} ; 1.2 \cdot C_{3}$ & 23.59 & 23.55 & 0.17 \\
\hline 20 & $0.8 \cdot C_{2} ; 0.8 \cdot C_{3}$ & 28.812 & 29.09 & 0.96 \\
\hline 21 & $1.2 \cdot C_{2} ; 0.8 \cdot C_{3}$ & 39.144 & 39.2 & 0.14 \\
\hline 22 & $0.8 \cdot C_{2} ; 1.2 \cdot C_{3}$ & 28.7 & 28.88 & 0.63 \\
\hline 23 & $1.2 \cdot R_{1} ; 1.2 \cdot C_{1}$ & 19.28 & 19.43 & 0.78 \\
\hline 24 & $0.8 \cdot R_{1} ; 0.8 \cdot C_{1}$ & 51.97 & 51.87 & 0.19 \\
\hline 25 & $1.2 \cdot R_{1} ; 0.8 \cdot C_{1}$ & 26.45 & 26.43 & 0.08 \\
\hline 26 & $0.8 \cdot R_{1} ; 1.2 \cdot C_{1}$ & 23.75 & 23.765 & 0.06 \\
\hline 27 & $1.2 \cdot R_{2} ; 1.2 \cdot C_{2}$ & 25.2 & 25.082 & 0.47 \\
\hline 28 & $0.8 \cdot R_{2} ; 0.8 \cdot C_{2}$ & 17.476 & 17.55 & 0.42 \\
\hline 29 & $1.2 \cdot R_{2} ; 0.8 \cdot C_{2}$ & 28.65 & 28.73 & 0.28 \\
\hline 30 & $0.8 \cdot R_{2} ; 1.2 \cdot C_{2}$ & 28.461 & 28.478 & 0.06 \\
\hline
\end{tabular}

clude now that the measurement error does not influence the diagnostics capacity of our ANN, which is one supplementary advantage of our method, and we can also conclude that extracting THD as the second parameter was a good choice.

\section{CONCLUSION}

Oscillation based diagnosis (OBD) has been introduced for the first time as a systematic method for diagnosis of analogue filter cells. The method was implemented on a second order Sallen and Key notch cell. A minimum number of test points and, accordingly, measurements were used: just the output terminal. The measured output signal was processed in order to obtain the following parameters: frequency of the first harmonic and total harmonic distortion. That is, in our opinion, a great advantage compared to ideas for the implementation of oscillation based testing requiring the supply current to be monitored. Single soft and catastrophic faults were considered in detail, while double soft faults are also shown to be detectable. The simulation before test concept was used to create fault dictionaries. The latter were memorized as parameters (weights and thresholds) of artificial 
neural networks. Diagnosis was performed by running the neural network after measurement (here simulation) of the faulty circuit. Noise was added to the signals obtained by simulation in order to check for the robustness of the method. It was shown that OBD may be successfully used for diagnosis of the notch cell, which in our experience is among the most difficult circuits to handle. In order to enable manipulation with an extremely large amount of data we intend to implement a hierarchical approach as it was done for testing purposes in [6].

\section{Acknowledgement}

This research was partly funded by The Ministry of Education and Science of Republic of Serbia under contract No. TR32004.

\section{REFERENCES}

[1] HURST, S.: VLSI Testing, Digital and Mixed Analogue/Digital Techniques, The Institution of Electrical Engineers, London, UK, 1988.

[2] ARABI, K.-KAMINSKA, B.: Oscillation-Test Strategy for Analog and Mixed-Signal Integrated Circuits, Proc. of the $14^{\text {th }}$ IEEE VLSI Test Symposium (VTS 96), Princeton, New Jersey, April/May 1996, pp. 476-482.

[3] ALLI, P. K.: Testing a CMOS Operational Amplifier Circuit Using a Combination of Oscillation and Iddq Test Methods, MSc Thesis, Louisiana State University, USA, 2004.

[4] HU, G.-WANG, H.-HU, M.-YANG, S.: Oscillation Test Strategy for Analog Filters by Monitoring Output Voltage and Supply Current, Thinghua Science and Technology 12 No. S1 (July 2007), 78-82.

[5] Litovski, V.-AnDREJEvić, M.-ZWOLInSKI, M. : Analogue Electronic Circuit Diagnosis Based on ANNs, Microelectronics Reliability, Elsevier 46 No. 8 (Aug 2006), 1382-1391.

[6] ANDREJEviĆ STOŠOviĆ, M.-MILOVANOVIĆ, D.-LITOVSKI, V.: Hierarchical Approach to Diagnosis of MixedMode Circuits using Artificial Neural Networks, Neural Network World, Institute of Computer Science AS CR, v.v.i. and Faculty of Transportation Sciences 21 No. 2 (2011), 153-168.

[7] ARABI, K.-KAMINSKA, B. : Efficient and Accurate Testing of Analog-to-Digital Converters using Oscillation-Test Method, Proc. of the European Design and Test Conference (ED\&TC 97), Paris, France, March 1997, pp. 348-352.

[8] ARABI, K.-KAMINSKA, B. : Oscillation-Test Methodology for Low-Cost Testing of Active Filters, IEEE Trans. on Instrumentation and Measurements 48 No. 4 (Aug 1999), 798-806.

[9] DAS, S. et al: Testing Analog and Mixed-Signal Circuits with Built-in Hardware - A New Approach, IEEE Trans. on Instrumentation and Measurement 56 No. 3 (June 2007), 840-855.

[10] HUElSMAN, L. P.: Active and Passive Analog Filter Design: An Introduction, McGraw-Hill College, 1993.

[11] LITOVSKI, V.-ZWOLINSKI, M.: VLSI Circuits Simulation and Optimization, Chapman and Hall, London, 1997.

[12] http://cds.linear.com/docs/Software\%20and\%20Simulation/ LTC6078-79.txt.

[13] MILIĆ, M.-ANDREJEVIĆ STOŠOVIĆ, M.-LITOVSKI, V. : Oscillation Based Analog Testing - A Case Study, $34^{\text {th }}$ International Convention on Information and Communication Technology, Electronics and Microelectronics - MIPRO 2011, Opatija, Croatia, 23-27 May 2011.

[14] CAI, J.-D.-YAN, R.-W.: Fault Diagnosis of Power Electronic Circuit Based on Random Forests Algorithm, Fifth IEEE International Conference on Natural Computation, Tianjin, China, 2009, pp. 214-217.
15] BENJAMINS, R.-JANSWEIJER, W.: Toward a Competence Theory of Diagnosis, IEEE Expert 9 No. 5 (1994), 43-52.

16] PIPITONE, F.-DEJONG, K.-SPEARS, W.: An Artificial Intelligence Approach to Analogue System Diagnosis, in Liu, R.-W., editor, Testing and Diagnosis of Analog Circuits and Systems, Van Nostrand Reinhold, New York, 1991, pp. 187-215.

[17] MAZUROVA, N. : Theoretical Background for the Applet-Based Exercises: Test Generation and Fault Diagnosis Boundary Scan, Tallinn Technical University, Faculty of Information Technology, Department of Computer Engineering, 2003, http://www.pld.ttu.ee/testing/theory/ LL-D\&T\%2BBS-lab_Mazurova.pdf.

[18] HAYASHI, S.-ASAKURA, T.-ZHANG, S. : Study of Machine Fault Diagnosis System using Neural Networks, Proc. of the Int. Joint Conf. on Neural Networks, Honolulu, Hawaii, 2002, pp. 233-238.

[19] GOLONEK, T.-RUTKOWSKI, J.: Use of Genetic Programming to Analog Fault Decoder Design, Proc. of the Int. Conf. on Signals and Electronic Systems, ICSES'02, Wrocław-Świerad 'w Zdrój, Poland, 2002.

[20] POUS, C.-COLOMER, J.-MELÉNDEZ, J.—de la ROSA, J. L.: Introducing Qualitative Reasoning in Fault Dictionaries Techniques for Analog Circuit Analysis, Sixteenth International Workshop on Qualitative Reasoning, Barcelona, Spain, 2002.

21] SCARSELli, F.-TSOI, A. C.: Universal Approximation using Feed-Forward Neural Networks: A Survey of some Existing Methods and some New Results, Neural Networks, Elsevier 11 (1998), 15-37.

[22] MANETTI, S.-PICCIRILLI, C. : A Singular-Value Decomposition Approach for Ambiguity Determination in Analog Circuits, IEEE Trans. on Circuits and Systems, I: Fundamental Theory and Applications 50 No. 4 (Apr 2003), 477-487.

23] BAUM, E. B.-HAUSSLER, D.: What Size Net Gives Valid Generalization, Neural Computing 1 (1989), 151-160.

[24] MASTERS, T.: Practical Neural Network Recipes in C++, Academic Press, San Diego, 1993.

Received 26 May 2011

Miona Andrejević Stošović was born in Niš, Serbia, in 1976. She graduated at the Faculty of Electronic Engineering, University of Niš, Serbia in 2000, at the department of Electronics. She received MS and PhD degree in 2003 and 2006, respectively, from the same faculty. Her research interests include neural networks based simulation and modeling for analog, digital and mixed-signal integrated circuits. She is the author of about 50 publications.

Miljana Milić was born in Bor, Serbia, in 1977. She graduated at the Faculty of Electronic Engineering, University of Niş, Serbia in 2001, at the department of Electronics. She received $\mathrm{MS}$ and $\mathrm{PhD}$ degrees in 2005 and 2009, respectively, from the same faculty. Her research interests include electronic circuit design and electronic circuit testing for analog, digital and mixed-signal integrated circuits. She is the author of about 50 publications. She received several rewards for her scientific research.

Vančo Litovski was born in Rakita, South Macedonia, Greece, in 1947. He graduated at the Faculty of Electronic Engineering, University of Niš, Serbia in 1970. He received MS and PhD degree in 1974 and 1977, respectively, from the same faculty. He is founder and President of the Yugoslav Simulation Society. He is member of The Academy of Engineering Sciences of Serbia and Montenegro, and is an IEEE member and author of about 400 publications. He received several awards for his scientific and educational work. 